\title{
Experimental Investigation of Contact Angle and Quench Severity of Mineral Oil and Palm Oil Blends
}

\author{
Peter Fernandes ${ }^{1}$, K. Narayan Prabhu ${ }^{2}$ \\ 1. Department of Mechanical Engineering, NMAM Institute of Technology, P.O. Nitte, 574110, Karnataka, India \\ 2. Department of Metallurgical \& Materials Engineering, National Institute of Technology Karnataka, Surathkal, P.O. \\ Srinivasnagar 575025, Karnataka State, India
}

Received: May 30, 2012 / Accepted: July 01, 2012 / Published: November 10, 2012.

\begin{abstract}
Wetting behavior and quench severity at the metal/quenchant interface would be useful to the heat treating community. In the present work, wetting behavior and the heat transfer characteristics of mineral oil and palm oil with their blends was determined. The heat transfer coefficients at the metal/quench medium interface were estimated of standard 304 stainless steel quench probes subjected to lateral quenching. The quench severity of vegetable oil is greater than conventional mineral oil with greater wettability indicated by its lower contact angles. Higher values of activation energy indicate a greater temperature dependency of contact angle relaxation. The relaxation of contact angle was sharp during the initial stages, and it became gradual as the system approached equilibrium. Blending of palm oil with mineral oil increases the spreading rate as well as the quench severity. This would have immense benefits from the environmental and economical point of view.
\end{abstract}

Key words: Heat transfer, heat transfer coefficients, quench severity, activation energy, wetting, blending.

\section{Introduction}

Heat treatment is defined as heating a metal to a specified temperature and holding at this temperature for required time followed by controlled cooling [1]. Steels may exhibit a wide variety of properties depending on the composition, phases and micro constituents [2]. Quenching of steel in heat treatment consists of raising the temperature of steel above austenitising temperature usually in the range of 850-870 ${ }^{\circ} \mathrm{C}$ holding it at that temperature for a fixed time and then rapidly cooled in a suitable quench medium to room temperature. The resulting properties from quenching depend on cooling rate and characteristics of steel. Successful quenching depends on the cooling characteristics of the quenching medium and the ability of steel to harden. The mechanism of heat removal during quenching is in three stages [3].

Corresponding author: Peter Fernandes, Principal, research fields: heat transfer, biofuel, wetting kinetics, composite materials. E-mail: petfdcm@gmail.com,petfcm@yahoo.co.in.
In the first stage, as soon as a work-piece comes in to contact with a liquid medium, the surrounding liquid layer is instantaneously heated up to the boiling point and gets vapourised and forms the vapour blanket. This vapour film is a poor conductor of heat and cooling of work-piece takes place by conduction and radiation through the vapour film and this stage is referred as the vapour blanket stage. The stable vapour film collapses and quenchant comes into contact with the hot metal surface causes nucleate boiling and resulting in higher heat extraction rates. As cooling continues, the surface temperature is reduced and metal surface is completely wetted by the liquid resulted in low cooling rate [4].

Quench severity refers to the ability of a quenchant or a quenching system to extract heat from a sample during quenching. Tensi and Lainer [5] studied rewetting and heat transfer during immersion quenching of high-speed quenching oils. The wettability of a surface was quantified by measuring "wetting" times. Wettability can be characterized by 
the degree and the rate of wetting or rate of spreading [6-7]. Contact angle is widely used to measure the wettability of liquid on the solid surface. It is an important parameter in surface science. Contact angle measurement provides a simple and reliable technique for the interpretation of surface energies [8]. The contact angle made by the liquid is the measure of the extent to which the liquid spreads on the solid substrate [9-10]. The basic mathematical treatment of wetting of a solid surface by a liquid is given by Young-Dupre equation which assumes equilibrium of interfacial energies and also gives an expression for contact angle $(\theta)$ formed at the three phase contact point.

The balance of interfacial energies under equilibrium gives[11-12] $\cos \theta=\left(\gamma_{\mathrm{sv}}-\gamma_{\mathrm{sl}}\right) / \gamma_{\mathrm{lv}}$, here ' $\gamma$ ' represents surface energy and subscripts $\mathrm{s}, 1$ and $\mathrm{v}$ indicate solid, liquid and vapour phases respectively. The rate of wetting indicates how fast the liquid spreads on the surface. Generally during spreading two forces that drive spreading capillary and gravity. The initial part of spreading curve is capillarity regime and followed by gravity. Effects of viscous flows can be incorporated into the molecular kinetic approach directly, by adding a viscous contribution to the barriers created by the liquid/solid attractions [13]. Quenchants can be divided into water, polymer, oils, salts and gases with different cooling characteristics which can be modified by variation of temperature, flow velocity and concentration [14].

The most commonly used oil is petroleum based quenchants due to its heat extraction characteristics. Mineral oil based products are used between $75 \%$ and $90 \%$ of all quenching operations. But petroleum based products are nonrenewable and can contribute to air and water pollution. The need to achieve energy independence and high cost of the crude petroleum is directing so much focus on vegetable oils and crops that will help to yield these oils. The vegetable oils are fully biodegradable hydraulic fluid, hence cause no pollution to water or soil and no toxicity to living organisms[15]. A bioquechant can be defined as a domestic, renewable quench medium derived from vegetable sources like sunflower, coconut, palm and groundnut [16].

\section{Experiments}

The experimental set-up for the quenching treatment consisted of a vertical tubular electric resistance furnace open at both the ends. A quench tank containing the quenching medium was placed directly underneath the furnace so that the heated workpiece could be transferred quickly to the quench medium. Quench probes were instrumented with K-type thermocouples of $0.45 \mathrm{~mm}$ diameter and were connected by means of compensating cables to a data-logger interfaced with a PC. A data logger (NI SCXI 1000) was used for acquisition of temperature data during quenching. The temperature data acquired during quenching of specimen was used as an input for calculating heat transfer coefficients.

Cylindrical quench probe having $12 \mathrm{~mm}$ diameter and $60 \mathrm{~mm}$ height was prepared from Type 304 stainless steel for assessment of quench severity and the metal/quenchant interfacial heat transfer. The surface of the probe was polished to 400 grit size after each test to ensure a similar surface condition. The dimensions of probe and the location of thermocouple (TC) are given in Fig. 1.

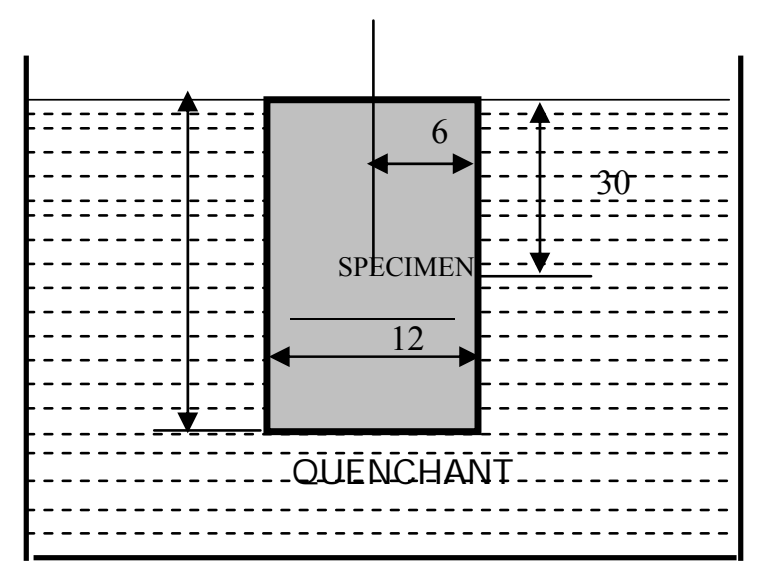

All dimensions are in $\mathrm{mm}$

Fig. 1 Schematic sketch of quenching set-up. 
The probes were heated to about $850{ }^{\circ} \mathrm{C}$ in the furnace and held vertically inside the furnace using a nichrome wire for 10 minutes and were quickly transferred to the quench tank containing $2000 \mathrm{ml}$ of quench medium. The temperature data acquired during quenching of specimen was used as an input to the lumped heat capacitance model for estimating heat transfer coefficients. The experimental set-up for the wetting study consists of a dynamic contact angle analyzer (Model: FTA 200, First Ten Angstroms, Virginia, USA). The equipment is capable of capturing and analyzing the spreading process of a liquid on a solid substrate.

The equipment has a flexible video system for measuring the contact angle, surface and interfacial energies. A droplet of test liquid was dispensed using a surgical syringe with a precision flow control valve on to the steel substrate and spreading phenomena was recorded at 60 images per second. Captured images were analyzed using FTA software to determine the contact angle of the droplet on the substrate.

Mineral oil (SN 150), palm oil and 25\% and 50\% blend of palm oil with mineral oil were used as test liquids for wetting studies on stainless steel (type 304) substrate and quench media for heat transfer studies.

\subsection{Assessment of Heat Transfer Coefficients}

The lumped heat capacitance method can efficiently model the unsteady cooling of a small object with an assumption of uniform probe temperature during quenching process[17-18]. If the temperature of the probe is uniform during cooling process $(\mathrm{Bi}=\mathrm{hd} / \mathrm{k}<0.1$ where $\mathrm{Bi}$ is the Biot number, $\mathrm{d}$ is the characteristic length (V/A) of the probe, and $\mathrm{k}$ is the thermal conductivity of the probe material (type 304 stainless steel) the heat loss from the probe ' $Q$ ' is equal to the decrease in internal energy of the probe. Thus

$$
Q=h A\left(T_{p}-T_{q}\right)=-C_{p} \rho V\left(\frac{d T_{p}}{d t}\right)
$$

where

$Q$ is the heat flow rate, $\mathrm{W} ; h$ is heat transfer coefficient on the probe surface, $\mathrm{W} / \mathrm{m}^{2} \cdot \mathrm{K} ; A$ is surface area of the probe, $\mathrm{m}^{2} ; \mathrm{T}_{p}$ is temperature of the probe, $\mathrm{K}$;
$T_{q}$ is temperature of the quenchant, $\mathrm{K} ; C_{p}$ is specific heat of the probe material, $\mathrm{J} / \mathrm{kg} \mathrm{K} ; \rho$ is specific density of the probe material, $\mathrm{kg} / \mathrm{m}^{3} ; V$ is volume of the probe, $\mathrm{m}^{3} ; t$ is time, $\mathrm{s} ;\left(d T_{p} / d t\right)$ is the cooling rate of the probe.

If the quenchant temperature around the probe $T_{q}$ is uniform, the above equation is rearranged as:

$$
Q=h\left(T_{p}-T_{q}\right)=-C_{p} \rho \frac{V}{A}\left(\frac{d T_{p}}{d t}\right)
$$

The heat transfer coefficient $(\mathrm{h})$ can be calculated from the cooling rate $\left(d T_{p} / d t\right)$ using the equation:

$$
h=C_{p} \rho \frac{V}{A}\left(\frac{d T_{p} / d t}{\left(T_{p}-T_{q}\right)}\right)
$$

Preciseness of $h$ depends on the accuracy of the cooling rate obtained from cooling curve data.

\section{Results and Discussion}

A typical thermal history during lateral quenching of Type 304 stainless steel probe in different oils with their blend is shown in Fig. 2. The three stages of quenching namely vapour blanket, nucleate boiling and convective cooling stages were observed in all quench media. Cooling rate was significantly higher during nucleate boiling stage in all quench media. Vegetable (palm) oil showed higher cooling rates compared to mineral oil.

Peak cooling rate of palm $\left(72^{\circ} \mathrm{C} / \mathrm{s}\right)$ was higher than mineral oil $\left(57{ }^{\circ} \mathrm{C} / \mathrm{s}\right)$. Among blend oils, highest peak cooling rate of $65^{\circ} \mathrm{C} / \mathrm{s}$ was obtained for $50 \mathrm{~m}: 50 \mathrm{p} \mathrm{(50 \%}$ mineral \& 50\% palm oil) blend oil whereas $75 \mathrm{~m}: 25 \mathrm{p}$ oil showed a lowest peak cooling rate of about $61^{\circ} \mathrm{C} / \mathrm{s}$.

Fig. 3 shows the variation of heat transfer coefficients (h) estimated by the lumped heat capacitance method with time during lateral quenching of stainless steel probe (Type 304) in different quench media.

High peak heat transfer coefficients were obtained for palm oil as compared to other oil media. Palm oil showed the maximum peak heat transfer coefficient $\left(770 \mathrm{~W} / \mathrm{m}^{2} \mathrm{~K}\right)$ and mineral oil showed the minimum peak heat transfer coefficient $\left(640 \mathrm{~W} / \mathrm{m}^{2} \mathrm{~K}\right)$. Peak heat transfer coefficient increased with the blend of palm oil 


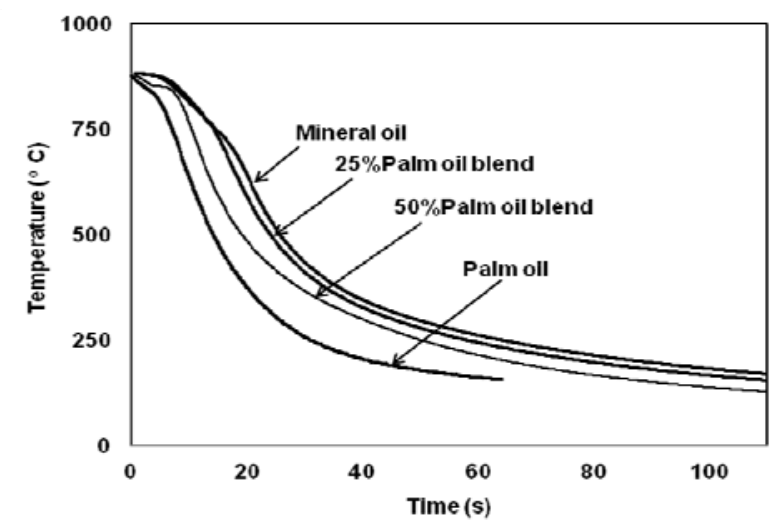

Fig. 2 Typical cooling curve during quenching of stainless steel probe in different quench media.

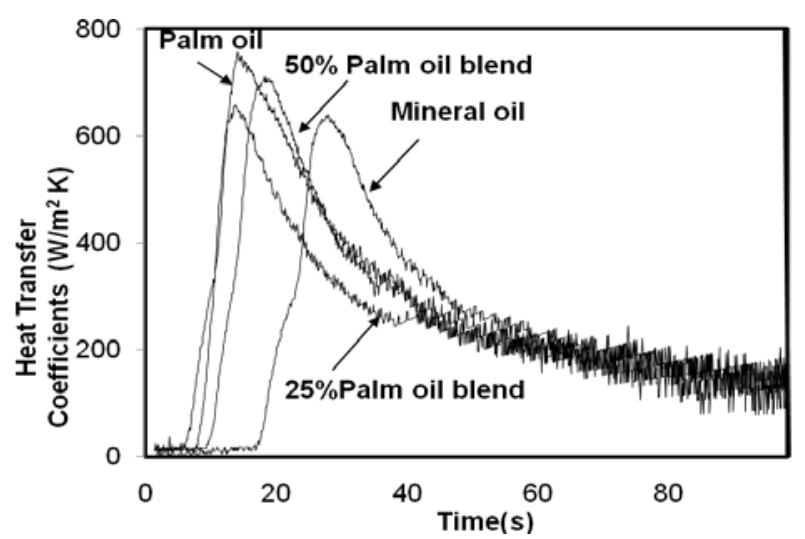

Fig. 3 Variation of heat transfer coefficients (h) with time during quenching of stainless steel probe in different quench media.

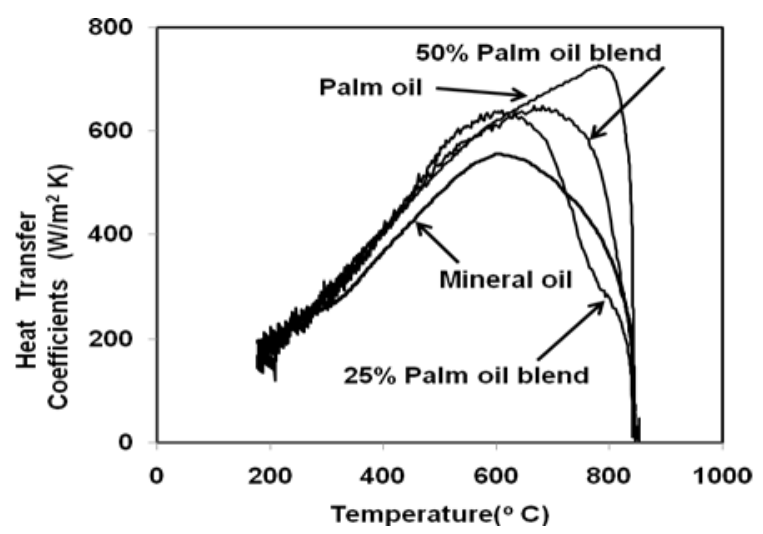

Fig. 4 Variation of heat transfer coefficients (h) with temperature during quenching of stainless steel probe in various quench media

with mineral oil. Variation of heat transfer coefficient (h) with temperature is shown in Fig. 4.

Peak heat transfer coefficients occurred at higher temperatures in palm oil than mineral oil. Similar results were obtained with blend of vegetable and mineral oil. The heat transfer coefficients were low in the initial period of quenching due to the insulating effect of the vapour blanket for all quench media. The duration of the existence of vapour blanket stage was more for mineral oil as compared with other medium. Therefore the nucleate boiling stage was earlier in palm oil compared with other medium. Heat transfer coefficients attained its maximum value during the beginning of nucleate boiling stage for all quench media. There is significant change in heat transfer coefficients occurs as increase in the percentage of palm oil in mineral oil.

Photographs of the droplets of quench media resting on the stainless steel substrate and measured contact angles for palm and mineral oil are shown in Fig.5. Fig. 6 shows the relaxation of contact angles of different oil media on stainless steel substrate at room temperature.

Relaxation of contact angle was rapid during early stages and it became gradual as the system approached equilibrium. Higher contact angles were obtained for mineral oil as compared with other oils. Highest equilibrium contact angle $\left(32^{\circ}\right)$ was obtained for

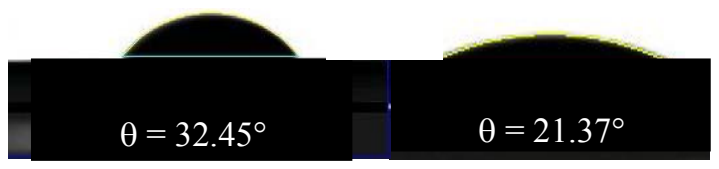
(a)Mineral oil
(b) Palm oil

Fig. 5 Images of quenchant droplets on stainless steel substrate.

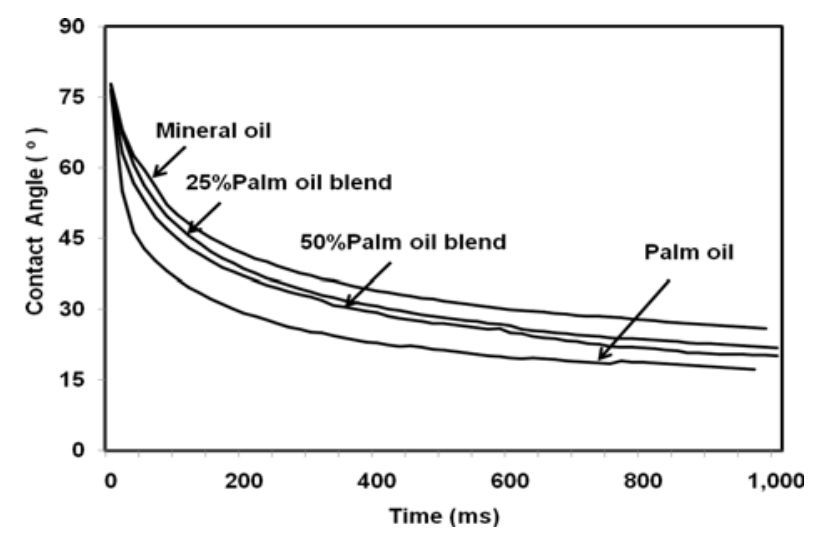

Fig. 6 Relaxation of contact angles of different quenchants on the stainless steel substrate. 
mineral oil and lowest equilibrium contact angle $\left(17^{\circ}\right)$ was obtained for palm oil on a stainless steel substrate. Contact angles decrease with increase in percentage of palm oil in mineral oil. Fig. 7 shows plot of Spread area Vs Time for different blend oils and mineral oil.

Low spreading was observed in mineral oil as compared to oil media. Higher spread area is the indication of the spreading of liquid drop during relaxation resulting in lower contact angle. Palm oil started spreading rapidly with a relatively high spread velocity resulting in a sharp increase of the base area during early stages of spreading. However, the spreading rate significantly reduced to almost zero indicating the condition of stabilization. This is due to the attainment of equilibrium between the various surface forces under action. Contact angles significantly decreased during spreading of oil media. The effect of temperature on equilibrium contact angle of oil media is shown in Fig. 8. It clearly shows that the equilibrium contact angle decreases with increase in the temperature of the substrate. Effect of substrate temperature is significant during the spreading of liquid medium.

The relaxation of contact angle was very rapid and the initial images of the droplet could not be recorded.

It was assumed that when $t=0$ the contact angle of the droplet is $180^{\circ}$ for all oils. The time required to reach $25^{\circ}$ was measured and the rate of contact angle relaxation was computed for all the oil media at temperatures varying from $30^{\circ} \mathrm{C}$ to $150^{\circ} \mathrm{C}$.

The variation of contact angle relaxation rate with the reciprocal of absolute temperature for various oil media is shown in Fig. 9. Contact angle relaxation rate was higher for all the oil media at higher temperatures.

The variation of rate of contact angle relaxation with temperature could be described by an Arrhenius type of Eq. (1) as:

$$
\frac{d \theta}{d t}=A \exp \left(\frac{-E_{a}}{R T}\right)
$$

where $d \theta / d t$ is rate of contact angle relaxation. $E_{a}$ is activation energy of the liquid, $\mathrm{J} / \mathrm{mol}, R$ is gas constant, $8.314 \mathrm{~J} / \mathrm{mol} \cdot \mathrm{K}, T$ is the absolute temperature, $\mathrm{K}$.

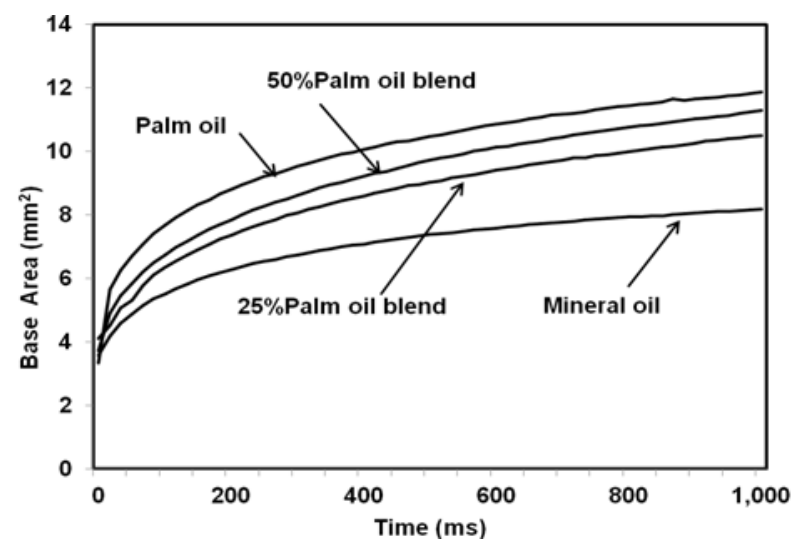

Fig. 7 Variation of base area with time during spreading of quench media on stainless steel substrate.

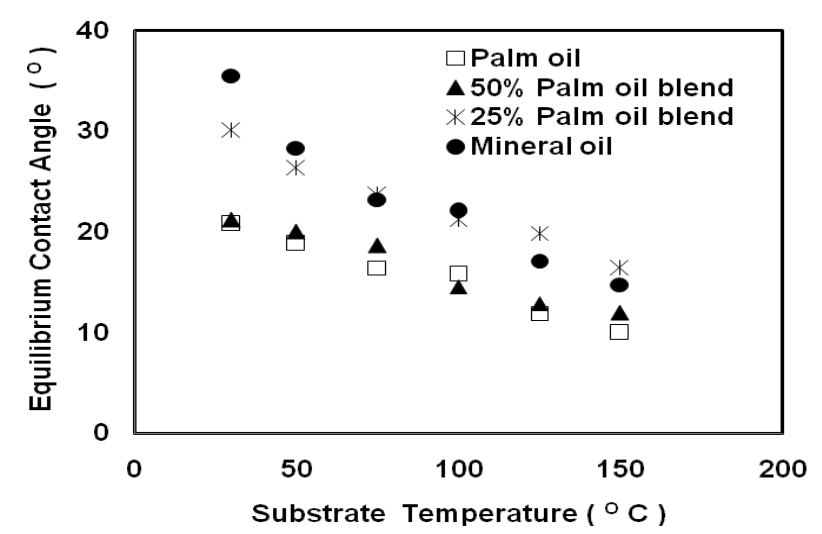

Fig. 8 Effect of substrate temperature on equilibrium contact angle of oil media.

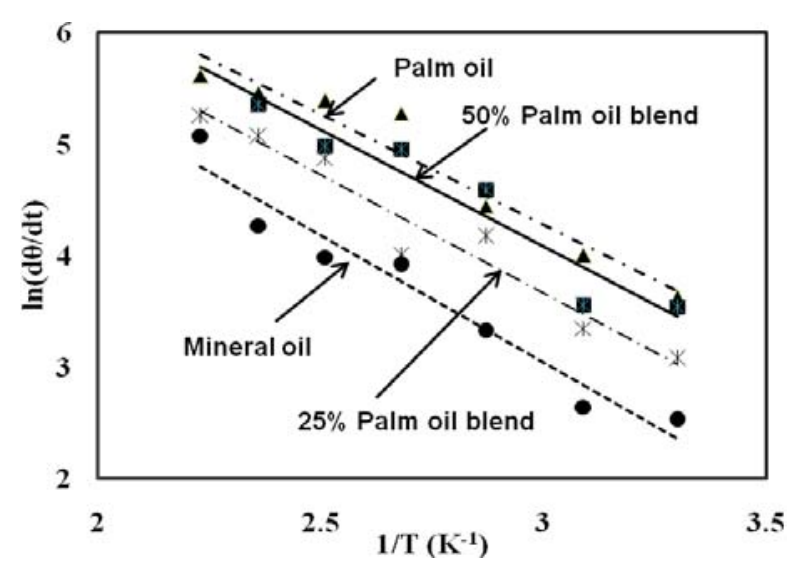

Fig. 9 Arrhenius plot of spreading of mineral oil and blend oils.

The Eq. (1) can be rewritten as:

$$
\ln \left(\frac{d \theta}{d t}\right)=\left(\frac{-E_{a}}{R}\right) \frac{1}{T}+\ln A
$$

A plot of $\ln (\mathrm{d} \theta / \mathrm{dt})$ Vs. 1/T yields a straight line of the type:

$$
y=m x+b
$$


where

$$
\begin{aligned}
& y=\ln (d \theta / d t) \\
& m=-E_{a} / R \\
& x=1 / T \\
& b=\ln A
\end{aligned}
$$

From the slopes of best fit straight lines (Fig. 9), the activation energy for spreading was computed. Increase in temperature increased the rate of relaxation. Higher values of activation energy indicate a greater temperature dependency of contact angle relaxation. Mineral oil showed the maximum activation energy of $22.7 \mathrm{~kJ} / \mathrm{mol}$ compared to other oils. It was observed that the palm oil has the lowest activation energy of $16.9 \mathrm{~kJ} / \mathrm{mol}$. Activation energy of $25 \%$ blend and $50 \%$ blend of palm oil is $18.9 \mathrm{~kJ} / \mathrm{mol}$ and $17.3 \mathrm{~kJ} / \mathrm{mol}$ respectively. Activation energy plays an important role in the spreading of oil media. A higher degree of spreading was observed in lower activation energy palm oil as compared to mineral oil with higher activation energy. Spreading of palm oil was terminated early as compared to mineral oil. Intermediate behavior was observed for blending of palm oil in mineral oil. The values of constant "ln A" were in the range of 9.8-11 for all oil media.

Fig. 10 shows $\ln$ (Base Radius)Vs $\ln$ (Time)for mineral and palm oil blends during spreading on stainless steel substrate. Among all oils, palm oil shows the higher base radius as compared to mineral oil and its blend. The spreading behaviour of various oils on the stainless steel substrate consisted of three stags, namely, capillary(a), gravity(b) and viscous regimes(c)[16]. Capillary force is the dominating force during initial capillary stage of the spreading of liquid. Capillary action is the ability of a substance to draw a liquid against the force of gravity. Gravitational force is the deciding factor in gravity regime. Viscous force is the dominating force, to cease the flow of liquid due to intermolecular resistance in viscous regime. The spreading begins with an initial capillary regime followed by a gravity regime and ends in viscous regime. It is clearly seen that, the spreading did not terminate early in palm oil and it was still in the gravity regime with nearly a constant slope. All the oils investigated exhibited the similar behaviour although the extent of spreading, indicated by their equilibrium contact angles, was different for different liquids.

The Rockwell hardness data obtained for AISI 1040 quenched steel specimens with different quench media are in the Table 1. Quenched specimens in Palm oil (lower contact angle) showed higher hardness compared to that obtained with other quenched samples. It is interesting to note that the $25 \%$ blend of palm oil with mineral oil which yielded the higher contact angle resulted in quenched specimens having lower values of hardness compared to that obtained with $50 \%$ blend of palm oil. The vegetable oil is thus safer compared to the mineral oil and could be exploited for industrial heat treatment.

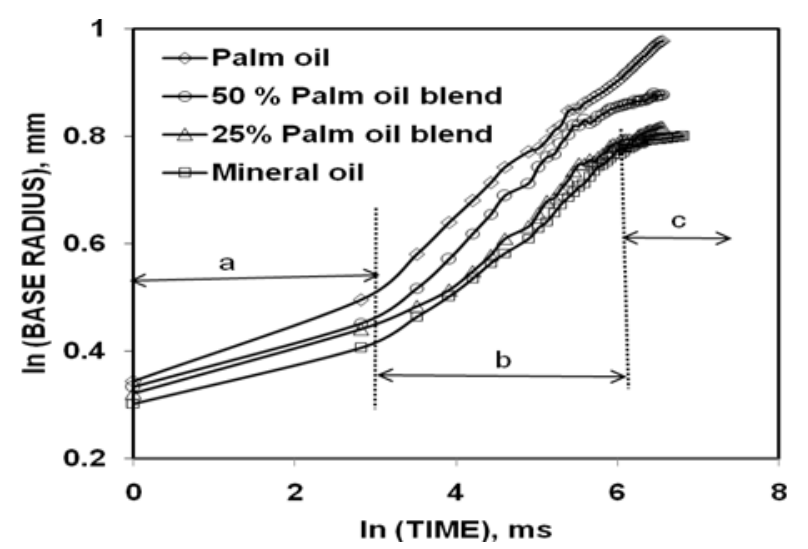

Fig. 10 Spreading of different oil media on stainless steel

\begin{tabular}{|c|c|c|c|c|}
\hline Sl. No. & Quench Media & Final Contact Angle $\left(^{\circ}\right)$ & Peak heat transfer coefficient $\left(\mathrm{W} / \mathrm{m}^{2} \mathrm{~K}\right)$ & $\mathrm{HRC}$ \\
\hline 1 & Palm oil & 17 & 770 & 51 \\
\hline 2 & Mineral oil & 32 & 640 & 42 \\
\hline 3 & $25 \%$ blend of Palm oil & 27 & 672 & 45 \\
\hline 4 & $50 \%$ blend of Palm oil & 25 & 716 & 48 \\
\hline
\end{tabular}
substrate showing (a) capillary (b) gravity (c) viscous regimes.

Table 1 Heat transfer and Wetting Data with Hardness. 


\section{Conclusions}

Based on the results of the investigation following conclusions were drawn.

(1) The contact angle relaxation of quench medium was rapid in the early stages of spreading and became gradual as equilibrium was approached.

(2) Among oils, palm oil showed higher spreading as compared to mineral oil and blend oils.

(3) High heat transfer coefficients were obtained with palm oil whereas conventional mineral oil yielded lowest heat transfer coefficients. Quench severity of vegetable oil blends was better than conventional mineral oil.

(4) Activation energy decreases with the increase in the percentage of palm oil in mineral oil.

(5) Blending of palm oil with mineral oil increases the spreading rate as well as the quench severity.

(6) For all quench media, higher rate of heat transfer was associated with higher wettability of the medium.

(7) Analysis of heat transfer and hardness data showed that cooling power of palm oil and its blending with mineral oil is better compared to pure mineral oil.

\section{References}

[1] American Society for Microbiology (ASM) Handbook, Heat treatment, ASM International, $10^{\text {th }}$ edition, Materials Park, OH, USA, Vol. 4, 1991, pp. 67-120.

[2] G.E. Totten, C.E. Bates, N.A. Clinton, Handbook of Quenching and Quenching Technology, American Society for Microbiology International, Materials Park, OH, USA, 1993, pp. 1-160.

[3] B. Liscic, State of the art in quenching, in: Proceedings of the 3rd Seminar of the International Federation of Heat Treatment and Surface Engineering, The Institute of Materials, London, 1993, pp.1-32.

[4] C. Tszeng, P. Nash, Modeling heat treating process, Industrial Heating, 68 (2001) 12-14.

[5] H.M. Tensi, K. Lanier, G.E. Totten, G.M. Webster, Quenching uniformity and surface cooling mechanisms, in: Proceedings of the 16th ASM, Heat Treating Society Conference and Exposition, Cincinnati, Ohio, 1996, pp. 3-8.
[6] S.G. Kandlikar, M.E. Sterinke, Contact angles and interface behaviour during rapid evaporation of liquid on a heated surface, International Journal of Heat and Mass Transfer, 45 (2006) 3771-3780.

[7] T.D. Blake, Y.D. Shikhmurzaev, Dynamic wetting by liquids of different viscosity, Journal of Colloid and Interface Science, 253 (2002) 196-202.

[8] C.N.C. Lam, N. Kim, D. Hui, D.Y. Kwok, M.L. Hair, A.W. Neumann, The effect of liquid properties to contact angle hysteresis, Colloids and Surfaces A 189 (2001) 265-278.

[9] T.D. Blake, J.D. Coninck, The influence of solid-liquid interactions on dynamic wetting, Advances in Colloid and Interface Science 96 (2002) 21-36.

[10] S. Sikalo, C. Tropea, E.N. Ganic, Dynamic wetting angle of a spreading droplet, Experimental Thermal and Fluid Science 29 (2005) 795-802.

[11] Y. Gu, D. Li, A model for a liquid drop spreading on a solid surface, Colloids and Surfaces A 142 (1998) 243-256.

[12] J. Long, M.N. Hyder, R.Y.M. Huang, P. Chen, Thermodynamic modeling of contact angles on rough, heterogeneous surfaces, Advances in Colloid and Interface Science 118 (2005) 173-190.

[13] J. DeConinck, M.J. DeRuijter, M. Voue, Dynamics of wetting, Current Opinion, Colloid and Interface Science, 6 (2001) 49-53.

[14] S. Sogerberg, E. Troell, The consequences of changing to alternative quenchants in heat treatment-materials, equipment and facilities, safety and environment, in: Proceedings of the 16th ASM Heat Treating Society Conference and Exposition, Cincinnati, Ohio, 1996, pp. 367-371.

[15] W.B.W. Nik, F.N. Ani, H.H. Masjuki, Thermal stability evaluation of palm oil as energy transport media, Energy Conversion and Management 46 (2005) 2198-2215.

[16] P. Fernandes, K.N. Prabhu, Comparative study of heat transfer and wetting behavior of conventional and bioquenchants for industrial heat treatment, International Journal of Heat and Mass Transfer 51 (2008) 526-538.

[17] X. Chen, L. Meekisho, G.E. Totten, Computer aided analysis of the quenching probe test, in: Proceedings of the 18th Conference of Heat Treating Symposium including the Lui Dai Memorial Symposium, ASM International, Materials Park, OH, 1998, pp. 601-609.

[18] A. Campo, J. Blotter, Experimental and numerical evaluation of the unsteady cooling of ball bearings in atmospheric air: Dual influence of nonlinear natural convection and surface radiation, Experimental Thermal and Fluid Science 23 (2000) 105-114. 